•综述・

\title{
植物生命之树重建的现状、问题和对策建议
}

\author{
王伟1,2* 刘 阳 ${ }^{3}$ \\ 1 (中国科学院植物研究所系统与进化植物学国家重点实验室, 北京 100093) \\ 2 (中国科学院大学, 北京 100049) \\ 3 (深圳市中国科学院仙湖植物园, 广东深圳 518004)
}

\begin{abstract}
摘要：生命之树的概念源自1859年达尔文的《物种起源》, 但利用分子数据重建植物生命之树的研究则在 20 世纪 90年代才开始兴起。近年来, 随着测序技术、分析方法和计算能力的快速发展, 植物生命之树重建研究取得了显 著成果。本文首先概述了当前以及未来很长一段时间内植物生命之树重建工作的重点，包括植物属级和种级水平 的系统发育研究、植物系统发育基因组学研究、分子和形态数据联合分析、包括灭绝与现存植物类群的生命之树 重建, 以及超大植物生命之树重建等5个方面; 然后简要概括国内植物生命之树重建研究的现状, 指出了我国在植 物生命之树重建领域发展中所存在的问题, 并从“类群研究体系、学科评价体系、国家顶层设计, 以及拓展国际合 作”等方面对学科末来的发展提出了一些对策建议。
\end{abstract}

关键词：分子系统学; 系统发育基因组学; 形态学; 超大树; 生命之树

\section{The current status, problems, and policy suggestions for reconstructing the plant tree of life}

\author{
Wei Wang ${ }^{1,2 *}$, Yang Liu ${ }^{3}$ \\ 1 State Key Laboratory of Systematic and Evolutionary Botany, Institute of Botany, Chinese Academy of Sciences, Beijing \\ 100093 \\ 2 University of Chinese Academy of Sciences, Beijing 100049 \\ 3 Fairylake Botanical Garden, Shenzhen \& Chinese Academy of Sciences, Shenzhen, Guangdong 518004
}

\begin{abstract}
In 1859, Charles Darwin put forward the concept of the tree of life (TOL), a metaphor for charting relationships between organisms in space and time in his The Origin of Species. The TOL is a cornerstone in evolutionary theory and makes sense of all biology. Decades of research in plant molecular systematics has led to substantial progress in understanding many aspects of the plant TOL. Here, we summarized five major aspects of reconstructing the plant TOL, which are being studied at the present day and will continue to be goals moving forward. These include: (1) constructing genus- and species-level phylogenies for plant groups; (2) resolving deep-time and/or rapidly divergent phylogenetic relationships using genomic approaches; (3) updating classification systems by combining morphological and molecular data; (4) integrating fossil taxa into phylogenies derived from extant taxa; and (5) building big trees using supermatrix methods. We then outlined the current state of plant molecular systematics and highlight existing problems in the field, specifically in regard to China. Finally, we propose the corresponding guidelines and policy suggestions for the continued study of China's reconstruction of the plant TOL.
\end{abstract}

Key words: molecular systematics; phylogenomics; morphology; big tree; tree of life

系统发育(phylogeny)的思想源自达尔文(Charles R. Darwin)的划时代著作—The Origin of Species (《物种起源》), 书中唯一的插图是一个代表物 种进化关系的系统发育树(phylogenetic tree)。达尔 文将生命之树(tree of life, TOL)视作系统发育的同 义词, 认为地球上所有的生物都起源于一个共同的

收稿日期: 2019-11-26; 接受日期: 2019-12-26

基金项目：中国科学院战略性科技先导专项 B 类(XDB31030000)和国家自然科学基金(31770233; 31770231; 31470315)

* 通讯作者 Author for correspondence. E-mail: wangwei1127@ibcas.ac.cn 
祖先, 它们之间的谱系关系可用一个树的形式来展 示, 即“the great tree of life”。生命之树上不仅有生 机勃勃的枝条, 也有死亡的枝条, 按照演化水平的 不同, 每一个物种在这棵树上都能找到它的位置 (Darwin, 1859)。鉴于地球上所有的现生物种通过其 祖先而享有共同的进化历史, 因此理解生物之间的 系统发育关系成为所有进化研究的先决条件。2013 年, 进化生物学专业期刊Systematic Biology上发表 了一首以“The tree of life”为题的讴歌生命之树的诗, 赞叹生命之树的伟大力量(Maddison, 2013)。生命之 树重建的目标是利用共同祖先遗传下来的性状构 建物种间真实的亲缘关系。在不同的分类群范围内， 根据物种性状的差异定义出门、纲、目、科、属和 种等分类等级, 代表了生命之树上的主干、分支和 末梢。重建一棵反映类群间系统发育关系的生命之 树可把分类学、古生物学、地学、分子生物学等学 科海量的信息融于一体, 不仅有助于人类理解生命 和各大门类生物的起源、演化及彼此之间的亲缘关 系, 而且能加深人们对现有生物多样性及其演化历 史和趋势的理解和认识(Benton \& Ayala, 2003)。

1866年，黑格尔著名的“树”的发表已体现了生 物学家对于系统发育概念的极大热情 (Haeckel, 1866)。但是, 直到20世纪60年代数量方法(numerical method) 出现之前, 人们通常只根据少数的形态性 状(如花部结构、果实类型、花粉性状、叶形及其表 皮解剖性状等)来推断生物的进化关系, 然而这样 得到的关系不能用其他的数据进行检验 (Nixon, 1996)。正如我们今天所认识到的, 不论是普遍流行 的，还是较有影响力的作者所构建的“系统发育关 系”, 只有正确的才能被广泛接受。例如, Takhtajan (1969)和Cronquist (1981)提出的被子植物的分类系 统虽然在分类学的背景下都是非常有用的, 但它们 现在已被认为是多个系统假设的集合，而这些假设 在很大程度上都是没有经过科学验证的。随着基于 共衍征(synapomorphy)的分支方法(cladistic methodology)的出现(Henning, 1950; Wagner, 1952), 系 统发育关系能被用程序化、可检验的分析方法所重 建, 从而建立的系统关系较为稳定。20世纪70年代, DNA测序技术的兴起使得人们能用蛋白质和DNA 序列来重建生物类群之间的系统发育关系。例如, Woese和Fox (1977)开创性地利用核糖体小亚基16S RNA的碱基序列构建了包括动物、植物和微生物的
生命之树。通过这棵树他们发现了古细菌域(Archaea), 并将生命世界划分为三个域: 真细菌、古细 菌、真核生物。相对于形态性状, 分子性状有3个明 显的优点: 可遗传、易确定同源性、能提供更丰富 的系统发育信息。因此，根据分子数据所建立的系 统发育关系更能真实地反映类群的进化历史。

2000年前后，分子系统学研究从使用单基因发 展到使用多基因，但通常少于 20 个基因(如Qiu et al, 1999; Moreira et al, 2000; Murphy et al, 2001)。 Bapteste等 (2002) 根据表达序列标签 (expressed sequence tag, 简称EST)数据获得了123个基因的蛋 白质序列, 据此重建了真核生物大类群间的系统发 育关系。这项工作开启了利用基因组数据重建生命 之树的先河。2003年, Eisen和Fraser (2003)正式提出 了系统发育基因组学(phylogenomics)的概念, 标志 着生命之树重建研究进入到一个崭新的时代一 系统发育基因组学时代(Delsuc et al, 2005)。植物分 子系统发育分析也经历了利用单基因、多基因，到 基因组数据的发展历程。1993年，Mark Chase等42 位作者合作发表了 “Phylogenetics of seed plants: an analysis of nucleotide sequences from the plastid gene $r b c L$ ”一文(Chase et al, 1993)。这是由世界几十个实 验室共同完成的当时规模最大的系统发育分析，是 植物生命之树重建研究领域一个里程碑式的工作。 从此之后, 人们广泛地利用DNA序列证据来重建植 物类群的系统发育关系, 植物分子系统学也作为植 物系统学的一个分支逐渐走向成熟。可以肯定地说, 由于分子系统学的兴起, 植物系统学在过去二十多 年所取得的成就远远超过了此前200多年所取得成 就的总和。这短短二十多年可看作是植物系统学, 甚至整个生物系统学发展的“黄金时代” (Soltis et al, 2009)。分子系统学现在不仅仅是一门学科, 已成为 生物学许多研究领域的一个有力工具。

\section{国际植物生命之树重建的发展示}

随着测序技术、分析方法和计算能力的飞速发 展, 国际植物生命之树重建在属级和种级水平的系 统发育研究、系统发育基因组学、分子和形态数据 联合分析、建立包括灭绝与现存植物类群的生命之 树, 以及超大植物生命之树重建等方面取得重大突 破, 成果显著。这 5 个方面也是当前以及未来很长一 段时间内植物生命之树重建工作的重点。 


\section{1 属级和种级水平的植物系统发育研究}

继Chase等(1993)大尺度的种子植物系统发育 分析之后, 基于单基因序列的多个被子植物大尺度 的研究连续发表。1998年, 被子植物系统发育组 (Angiosperm Phylogeny Group, 简称APG)成立, 并 综合之前的研究结果, 为被子植物提出了一个目、 科分类阶元上的分类系统，简称 APG 系统 (APG, 1998)。被子植物成为第一个基于分子数据建立分类 系统的大类群。之后, 随着基因和类群取样数量的 增加, APG系统经历了三次修订(王伟等, 2017)。其 他的植物大类群, 如裸子植物( Lu et al, 2014)和硕 类(Lehtonen, 2011)等目、科水平上的系统发育关系 也都得到了很好的解决。American Journal of Botany 在建刊90周年之际, 以“The Plant Tree of Life”为主 题组织专辑, 全面介绍了分子系统学在植物生命之 树重建方面取得的巨大成果(Palmer et al, 2004)。 2016年, Journal of Systematics and Evolution以“Systematics and Evolution of Lycophytes and Ferns”为主 题组织专辑, 从分类、系统发育到区域硕类的多样 性等方面对嵚类研究进行了报道(Schneider \& Schuettpelz, 2016)。

尽管植物界在目、科水平上有了一个比较清晰 的系统发育框架，但这仅是植物生命之树重建“万 里长征”完成的第一步。我们知道, 地球上的绿色植 物约有 500,000 种, 到目前为止仅有不足 $30 \%$ 的物种 有分子序列报道(Hinchliff et al, 2015; Folk et al, 2018)。即使在属级水平上, 也仍然有大量的工作需 要开展。比如, 药用和观赏价值较高的毛茛科约有 60 属, DNA数据分析结果表明该科 $1 / 3$ 的属的范围 或名称需要调整(Wang et al, 2013)。Kadereit等(2016) 对涉及德国维管植物的分子系统学研究进行了整 理, 发现在德国分布的840属中, 大约140属不是单 系, 且另外有20余属的单系性也可能存在问题, 需 要进一步研究。我们通过ISI Web of Science网站对 2005年以来发表的现生植物的新属进行了统计, 发 现自2008年起, 每年至少有25个新属被发表, 且绝 大多数集中在被子植物, 尤其是真双子叶植物中。 目前在各种国际分子系统学期刊, 如 Molecular Phylogenetics and Evolution、Taxon 和 Systematic Botany等, 每一期几乎都有植物属、种水平的系统 发育研究论文。属、种水平的系统发育研究将是植 物生命之树重建未来很长一段时间的工作重点。

\section{2 植物系统发育基因组学研究}

系统发育基因组学是进化生物学领域由系统 发育学和基因组学相结合形成的一门崭新的交叉 学科(Eisen \& Fraser, 2003)。基因序列是生物遗传信 息的载体，而基因组是遗传信息的总和。由于基因 组能提供更多的分子性状，系统发育基因组学在解 决疑难系统发育关系，如快速辐射类群的系统关 系、子遗类群的系统位置等方面得到了广泛应用。 植物叶绿体基因组一般为共价闭合环状结构, 具有 很好的共线性，几乎不存在(或很少受)旁系同源 (paralogy)基因的干扰，且在细胞中以多拷贝的形式 存在, 因此叶绿体基因组在植物系统发育基因组学 研究中首先得到了应用(张韵洁和李德铢, 2011)。第 二代测序(next-generation sequencing, 简称NGS)技 术的出现大大降低了叶绿体基因组测序的成本。该 技术通过增加测序深度(sequencing depth)并借助不 断优化的基因组组装算法，弥补了其测序有效读长 短、错误率较高的问题，从而能在短时间内同时获 得多个高质量的叶绿体基因组数据，且测序深度为 传统方法的2-10倍。Moore等(2006)首次应用454测 序平台, 对南天竹(Nandina domestica)和一球悬铃 木(Platanus occidentalis)进行测序, 两个叶绿体基 因组均获得了高于 $99.75 \%$ 的基因组覆盖度, 测序深 度分别为 $24.6 \times$ 和 $17.3 \times$ 。测序技术的突破大大促进 了叶绿体系统发育基因组学的发展。Jansen等(2007) 根据64个叶绿体基因组的 81 个基因解决了被子植 物最早分化的三个支系的系统发育关系，即无油樟 目是被子植物最早分化的支系, 随后依次是睡莲目 和木兰藤目。Moore等(2007)测得金鱼藻(Ceratophyllum demersum)的叶绿体基因组, 并结合其他44 种植物的叶绿体基因组，利用61个基因解决了金鱼 藻的系统位置。随后, Moore等(2010)进一步选取86 个种子植物叶绿体基因组的 83 个蛋白质编码基因 和rRNA基因，对被子植物做了比较全面的分析， 很好地解决了真双子叶植物 (eudicots) 内部的系统 发育关系，并发现超蓄薇类(superrosids)和超菊类 (superasterids)的主要支系均在约5百万年内快速出 现。这3个成果均刊登在国际主流期刊Proceedings of the National Academy of Sciences of of the United States of America (PNAS)上。

随着越来越多的叶绿体基因组被应用到植物 系统发育分析中，一些学者认识到仅仅把DNA序列 
进行联合分析可能会产生一些错误的结果, 尤其在 大尺度分析中。比如, Goremykin等(2013)在移除叶 绿体基因中进化速率较快的位点之后, 发现无油樟 目和睡莲目一起构成被子植物最早分化的支系, 这 与大家普遍接受的无油樟目独自为被子植物最早 支系的观点相矛盾。不过, Drew等(2014)发现 Goremykin等(2013)所移去的 “噪音” 数据也支持无 油樟目是被子植物最早分化的支系。在陆地植物系 统发育分析中, Cox等(2014)发现考虑叶绿体基因同 义密码子偏选和直接联合分析得到的结果存在显 著的冲突。除了考虑进化噪音对系统发育分析的影 响外，一些新的核苷酸替代模型或分析方法也被提 出。如Goremykin等(2013)提出了考虑碱基组成异质 性的模型 $($ CAT + GTR $+\Gamma+$ covext model $)$ 。在利用 82 个叶绿体基因对金虎尾目进行研究时, Xi等(2012) 提出了基于贝叶斯混合模型的后验数据分区(posteriori data partitioning)方法, 很好地解决了这个被 称为被子植物中最“顽拗支系” (recalcitrant clade)的 系统关系。

除了叶绿体基因组, 线粒体基因组也被用于植 物的系统发育分析。由于线粒体基因进化速率较慢, 通常用于较高分类阶元的系统学研究。如Liu等 (2014)组装了 20 个苔藓的线粒体基因组, 结合其他 已发表的 40 个植物的线粒体基因组, 利用 41 个线粒 体基因, 通过剔除系统误差, 包括进化饱和、谱系 间GC含量异质性、同义密码子偏选等, 重建了早期 陆地植物间的系统关系, 说明了在核苷酸序列分析 中进行序列简并测试以及使用更复杂的模型等方 法的重要性。

核基因组比细胞器基因组大, 又是双亲遗传, 不仅能提供更多的性状，而且可揭示网状进化事件， 因此在系统发育研究中具有更大的应用潜力, 可能 是未来植物系统学的主要发展方向。但由于核基因 组测序相对困难, 核系统发育基因组学起步稍晚。 最早人们通过已公布的EST数据或利用转录组 (transcriptome)测序技术来获得大量直系同源核基 因。如Lee等(2011)通过145种植物的EST数据以及 5 种植物的核基因组数据, 篮选出 2,970 个直系同源 基因, 在陆地植物框架下重建了种子植物较高分类 阶元的系统发育关系。利用1KP (千种植物转录组) 项目产生的数据, Wickett等(2014)选取92种陆地植 物及藻类, 使用 852 个核基因重建了陆地植物大尺
度的系统关系。在此基础上, 2019年10月, 千种植物 转录组联盟(One Thousand Plant Transcriptomes Initiative)利用 1,178 种植物的转录组数据, 在国际 主流学术期刊Nature 上发表了题为“One thousand plant transcriptomes and the phylogenomics of green plants”的成果(One Thousand Plant Transcriptomes Initiative, 2019)。

除了进化饱和、GC含量异质性、同义密码子偏 选等系统误差外, 不完全谱系分选 (incomplete lineage sorting)是核系统发育基因组学研究中遇到 的最大进化噪音, 它使得直接联合DNA或蛋白质序 列进行系统发育分析可能得到错误的结果。溯祖理 论(coalescent theory)可允许不同基因有不同的进化 历史, 从而排除不完全谱系分选对系统发育分析的 影响(Zhong et al, 2013; Liu et al, 2015), 近年来该方 法在核系统发育基因组学分析中得到越来越广泛 的应用。

对于核基因测序，由于转录组测序对实验材料 要求较高, 一些替代方法, 如简化基因组(restriction-site associated DNA sequencing, 简称RAD-Seq)、 靶向富集(target enrichment)、基因组浅层(genome skimming)等技术应运而生(McKain et al, 2018)。尤 其是靶向测序可以同时获得叶绿体、线粒体和细胞 核三套基因组的数据, 具有广阔的应用前景。自 2018年以来, 一些基于靶向测序方法的植物基因组 系统学研究已经陆续发表。例如, Medina等(2018) 使用靶向测序获得了全部质体基因序列, 解决了苔 藓植物葫芦藓科中辐射演化类群的系统关系; Morales-Briones等(2018)通过靶向测序获得了396个 核基因和全叶绿体基因序列, 构建了稳定的云衣草 属(Lachemilla)的系统关系; Liu等(2019)使用靶向测 序技术获得了 40 个线粒体基因、82个质体基因以及 150 个单拷贝核基因的数据, 为藓类植物建立了可 靠的目级水平的系统发育框架。同时, 植物全基因 组测序的发展也异常迅速。目前大约已有 700 种植 物的基因组被测序(www.ncbi.nlm.nih.gov/genome)。 随着测序技术的进一步发展和测序成本的降低, 快 速、批量测序植物基因组的时代正在来临。2015年, 在美国华盛顿国立博物馆举行的“首届全球生物多 样性基因组学” 会议上, 包括华大基因理事长杨焕 明、深圳国家基因库副主任张国捷等在内的23名科 学家提出了“地球生物基因组计划” (Earth 
BioGenome Project, EBP) (Pennisi, 2017)。该项目计 划用 10 年以上的时间, 对地球上包括植物在内的 150万种真核生物的基因组进行测序, 项目总计将 耗资47亿美元(Lewin et al, 2018)。作为对该计划的 响应，华大基因在2017年的第19届植物学大会上宣 布启动 1 万个植物基因组计划(10,000 Plant Genome Sequencing Project, 10KP) (Cheng et al, 2018; Twyford, 2018)。英国桑格研究所联合伦敦自然历史博 物馆、英国皇家植物园和爱丁堡大学等机构于 2018 年11月发起了达尔文生命之树计划(Darwin Tree of Life Project), 该计划声明将在10年内完成包括植物 在内的66,000种英国本土真核生物物种的基因组测序 (www.sanger.ac.uk/news/view/genetic-code-66000-uk -species-be-sequenced/)。同时，英国邱园于2018年启 动了植物-真菌生命之树项目 (Plant and Fungal Trees of Life, 简称PAFTOL), 该项目计划利用靶向 测序技术在 2020 年完成所有 14,000 个被子植物属和 所有 8,200 个真菌属的 353 个基因的测序工作，从而 重建其属级水平的生命之树。这些项目的开展必将 对植物生命之树的重建工作起到巨大的推动作用。

2015年, Journal of Systematics and Evolution发 表了以“Phylogenomic Approaches to Deciphering the Tree of Life”为主题的专辑(Wen et al, 2015)。2018年, American Journal of Botany和Applications in Plant Sciences分别发表了以“Using and Navigating the Plant Tree of Life” (Soltis et al, 2018)和“Methods for Exploring the Plant Tree Life" (Gitzendanner et al, 2018)为主题的专辑。这3个专辑通过综述和研究论 文的形式系统地总结了植物系统发育基因组学在 测序技术、分析方法和特定植物类群系统关系等方 面所取得的成果, 并探讨了当前面临的问题以及未 来可能的发展方向。

\section{3 分子和形态数据联合分析}

在分子系统学快速兴起之时, 形态学研究则相 对没落, 在某种程度上甚至被遗弃了。近年来, 超 过95\%的系统学文章都是仅仅使用了DNA序列数 据(见Soltis等(2018)组织的专辑)。系统与进化生物 学经历了一个多世纪的发展, 人们通过形态学、解 剖学、胚胎学、狍粉学、细胞学和个体发生学等方 面的研究积累了丰富的广义形态性状, 而DNA序列 仅为一类独立的性状, 分子和形态数据的结合能大 大增加我们追溯植物类群的系统发育关系与植物
演化过程的能力(Nandi et al, 1998; Doyle \& Endress, 2000)。以前, 最大简约法(maximum parsimony)是分 析形态和分子联合数据唯一可行的方法, 但它易受 长支吸引(long-branch attraction)影响(Swofford et al, 2001)。近年来, 新发展的运用统计进化模型的建树 方法一贝叶斯法(Bayesian inference), 可分析形 态数据或形态和分子联合数据, 大大提高了形态性 状的应用价值(Glenner et al, 2004; O’Reilly et al, 2018)。但是, 分析形态和分子联合数据时, 贝叶斯 法在动物研究中的应用比较普遍, 而在植物研究中 应用相对较少。

一个合理且方便使用的分类系统应是在系统 发育背景下先确定各支系的鉴别特征(diagnostic character), 然后根据鉴别特征提出的，而仅仅根据 分子证据提出的分类系统有时难以从形态学的角 度去理解, 且不方便使用。比如, APG分类系统所界 定的金虎尾目包括了 36 个科，这些科在Takhtajan (2009)的系统中被放在了 13 个目。从形态学的角度 看, APG的金虎尾目就是一个大“拼盘”。同时，由于 缺乏形态性状的分析, APG 分类系统既没有一个 目、科水平的检索表，也没有目、科的特征描述，从 而限制了它的广泛使用(王伟等, 2017)。Stull等(2018) 利用73个叶绿体基因构建了包括248 个物种的菊亚 纲的系统发育树，然后探讨了15个性状(包括植化、 解剖、花、果、种子等)的进化式样, 发现多个性状 可能是菊亚纲各主要支系的共衍征，同时，也指出 一些重要支系的性状仍然是不清楚的, 强调对那些 知之甚少的支系开展形态学研究是我们更好地理 解菊类植物早期进化历史的关键。对形态性状进行 编码可以帮助我们进一步探讨分子进化速率与植 物生活史之间的联系(Smith \& Donoghue, 2008), 或 者验证某些特殊性状的进化与物种多样化速率的 变化之间是否存在一定的相关性(Maddison et al, 2007)。2010年, 美国佛罗里达大学的Douglas E. Soltis教授在其被子植物生命之树(ATOL)项目中计 划建立MorphBank，其目标是建立一个包括15,000 种的 20 个形态性状的被子植物形态特征的标准图 谱, 希望MorphBank能成为古植物学家、植物分类 学家和生态学家一个可以实时利用的综合资源平 台。但很遗憾，这个项目并没有获得资助。

\section{4 包括灭绝与现存植物类群生命之树的研究}

一棵完整的生命之树不仅包括所有的现生类 
群，也包括了所有灭绝的类群。可是，当前超过 $95 \%$ 的系统发育分析都是仅针对现生类群的(见Soltis 等(2018)组织的专辑)。就目前技术而言，几乎不可 能从灭绝植物类群中获得核苷酸序列。因此, 形态 性状成为对化石植物类群进行系统发育分析的唯 一选择。适合分析形态性状的贝叶斯法的普及大大 加速了化石类群的系统发育分析(O'Reilly et al, 2018; Schrago et al, 2018)。与此同时, 古植物学家采 用新的技术和手段从化石中获取准确的形态性状。 比如, 扫描电镜(scanning electron microscopy, SEM) 和同步加速 X射线层析显微 (synchrotron radiation X-ray tomographic microscopy, SRXTM)技术已被广 泛应用到植物化石的研究中, 它们能在不损坏化石 的情况下获得化石内部的形态性状, 同时可分辨化 石的真伪。例如, Friis等(2016)用此技术报道了迄今 最早的核心真双子叶植物的化石。然而, 现生植物 类群中用这两个技术进行形态学研究的较少, 这限 制了整合化石和现生类群的系统发育分析。

整合化石和现生植物类群进行系统发育分析, 可以更为全面地理解形态性状的演化式样, 为分化 时间估算提供更为可靠的化石标定点, 有时还能有 助于解决植物类群之间的系统发育关系。Henning (1966)曾预言加入化石植物类群不会改变现存物种 之间的系统关系。但是, 一些研究发现加入化石类 群能改变仅仅根据现存物种建立的系统发育关系 (如Atkinso, 2018; Rothwell et al, 2018b)。2018年, American Journal of Botany发表了以“Tree of Death: The Role of Fossils in Resolving the Overall Pattern of Plant Phylogeny”为主题的专辑, 以实际案例阐述 了包括灭绝类群对系统发育分析可能的5种影响: (1) 加入到现生类群的系统树上, 增加了树的完整性, 但不改变整个树的拓扑结构; (2)明显改变了树的拓 扑结构; (3)由于缺少或者有冲突的系统发育信息, 使一些节点(node)变成多歧分支(ploytomy); (4)解决 了一些内部的多歧分支; (5)丰富了那些遭受大量灭 绝支系的系统树的拓扑结构, 并增加了系统树上物 种的丰富度(Rothwell et al, 2018a)。

在构建时间树方面, 化石类群通常作为限制时 间标定点的方式来使用, 如在BEAST软件下进行的 分化时间计算。对于这样的使用方式, 如果多个不 同时期的化石出现在同一支系上, 需选择最古老的 化石作为标定点, 删去其他咒余的化石。同时, 化
石类群参与生物地理分析常用的做法是将化石插 入到现生类群的系统树上，但如果多个同时期的化 石出现在相同的节点上，这种做法将产生多歧分支， 即出现零支长。近年来新发展的末端时间计算(tip dating)方法可把化石当作末端类群(terminal或tip), 利用形态或者形态和分子联合矩阵来计算分化时 间，产生包括化石在内的时间树(time tree) (Pyron, 2011)。这种方法能包括所有可以获得的化石类群参 与时间树的构建, 并避免出现零支长。这对化石分 布区不在现生类群分布区之内的生物地理学研究 尤为重要(Lavoué, 2016; Yonezawa et al, 2017; Xiang et al 2019)。目前，末端时间计算方法主要应用于动 物的研究, 而在植物研究中鲜有使用。

\section{5 超大植物生命之树重建的研究}

随着植物系统学的飞速发展, 海量的数据已经 产生并可从各种公共数据库下载, 如 NCBI (www.ncbi.nlm.nih.gov/)、TreeBASE (www.treebase. org/)和Dryad (www.datadryad.org/)等, 这为人们构 建超大系统发育树(mega-phylogeny)提供了可能。构 建超大系统发育树有两种方法: 超树(supertree)和 超矩阵(supermatrix)。超树是指先单独构建多个具 有重叠类群的“小树”，然后用这些“小树”整合得到 一棵 “大树”; 超矩阵是指通过具有重叠类群的分子 数据组装一个包括尽可能多的类群的超大矩阵, 进 而通过系统发育分析构建一个“大树” (Smith et al, 2009)。由于后者具有遗传信息，可进行进一步的信 息挖掘，比如分化时间的估算等，因此应用更为普 遍。对于生态学家而言，一棵系统树尽可能地包括 更多的物种比提高其支持率更重要。超大植物生命 之树主要应用于探讨生物多样性的形成过程和进 化动态。比如, Zanne等(2014)构建了一个包括32,223 种陆地植物的时间树，揭示了被子植物快速辐射到 寒冷生境中的三个关键创新：木本植物中出现了小 的导管和落叶的习性, 草本植物中发生了地上部分 快速衰老的现象。Smith等(2018)构建了包括5,036 个物种的石竹目的系统树, 发现多个多样化速率的 转变都发生在全基因组复制(whole genome duplication, WGD)之后。Folk等(2018)以蓄微类为例探讨了 构建全球尺度的物种水平的广泛取样的系统树，以 及与之相联系的地理分布和表型数据的机遇和挑 战。目前，利用特定类群的超大系统树探讨其多样 性的形成过程和机制方面发展迅速，被子植物中一 
些目、科以及较大的属已被分析，包括川续断目、 报春花目、禾本科、苦苣苔科、柳叶菜科、菖麻科、 楝科、桔梗科、荚蒾属(Viburnum)等, 这些工作都发 表在国际主流期刊上，如Science、PNAS、Systematic Biology、Ecology Letters、New Phytologist等。

同时, 也有一些研究利用超矩阵的方式构建了 区域植物生命之树的工作，进而探讨其系统发育多 样性和群落结构。如Kerkhoff等(2014) 以新世界 12,521种木本被子植物为研究对象, 通过构建时间 树, 整合物种分布和环境气候等信息, 揭示出新世 界大量的温带类群分支是渐新世以来由于全球温 度骤降而出现快速分化的, 其物种多样性的时空格 局符合热带保守性假说(tropical conservatism hypothesis)。Thornhill等(2017)构建了加利福尼亚植物区 系的生命之树, 探讨了该地区系统发育多样性、系 统发育特有性，以及古特有和新特有的特点。

准确分析超大数据矩阵是构建 “大树” 的前提, 也是当前面临的挑战，尤其是构建超大的时间树， 对计算机运算能力提出了更高的要求。利用图形处 理器(graphics processing unit, GPU)多核资源, 使用 计算机集群、云计算平台, 以及对分析软件进行并 行版本升级都已得到广泛的实践(Hamilton \& Buell, 2012)。iPlant科研团队正在开发能够用于大尺度的 系统发育分析的工具(www.iplantcollaborative.org/)。 随着建立的系统树越来越大，如何将所构建的“大 树”可视化也是一个亟待解决的问题。此外, 为科学 和大众提供一棵整合的、开放的、可实时更新的植 物生命之树将是未来一项巨大的挑战(Eiserhardt et al, 2018)。Hinchliff等(2015)已为此进行了探索, 构 建了一个包括2,300,000个类群的开放的生命之树。

\section{2 我国植物生命之树重建的发展}

Chen等采用银染测序方法历经两年完成了马 尾树 (Rhoiptelea chiliantha) 的 rbcL的序列测定, 并 于1998年发表了 “根据叶绿体 $r b c L$ 基因序列探讨马 尾树科的系统位置”一文(Chen et al, 1998)。这是中 国学者在国内实验室完成的最早的分子系统学论 文, 标志着我国植物系统学研究进入到分子时代。 2007年6月, 在北京召开了“生命之树国际学术研讨 会”, 极大提升了我国植物分子系统学的研究水平。 近年来, 我国植物生命之树重建领域发展迅速, 表 现出很强的创新能力和国际竞争力, 已经利用多基
因、分子和形态联合，以及基因组数据，在不同分 类阶元的植物类群的系统发育和区域生命之树研 究中取得了具有世界影响的成果。

在利用多基因数据方面, 我国科学家澄清了许 多疑难植物类群的系统位置和分类等级。例如, $\mathrm{Su}$ 等(2012)利用 4 个叶绿体DNA片段对十字花目的 12 科39属进行系统发育分析，发现节蒴木属(Borthwickia)不是山柑科的成员，进而结合形态证据将该 属提升至科的分类等级——节蒴木科(Borthwickiaceae)。这是由中国学者命名的第 4 个被子植物科级 分类单元。Wang等(2017)利用5个叶绿体和 1 个核的 DNA片段重建了防己科祝酒藤族的系统发育关系， 发现宽筋藤属(Tinospora)的物种隶属于 3 个不同的 支系，然后通过整合系统发育、分化时间和形态学 证据，将这3个分支界定为3个不同的属，包括建立 的新属——青牛胆属(Paratinospora)和重新恢复的 琉荌藤属(Hyalosepalum)。近年来, 利用分子证据, 我国植物系统学家已经重新界定了大量我国分布 的植物属, 包括硕类在内发表了许多新属, 如线翠 蕨属(Gastoniella)、假粗叶木属(Paralasianthus)、北 油丹属(Alseodaphnopsis)、露荵乌头属(Gymnaconitum)、纪如竹属(Hsuehochloa)、假合头菊属(Parasyncalathium)、希陶木属(Tsaiodendron)、征镒麻属 (Zhengyia)、征镒木属(Wuodendron)等，其中不少是 中国特有属, 比如露荵乌头属、征镒麻属和青牛胆 属等。同时，也发现了大量的新种和新记录属、种。 这些工作不仅提高了人们对这些类群进化历史的 认识, 而且大大丰富了我国的植物多样性。尤为重 要的是, 我国植物学家对植物的各个重要代表类群, 从低等藻类到高度进化的被子植物, 尤其是东亚分 布为主的科、属开展了系统而深入的研究, 建立和 完善了众多植物类群的系统发育关系, 相关成果发 表在Cladistics、Molecular Phylogenetics and Evolution、Taxon、Botanical Journal of the Linnean Society 等进化领域主流期刊上，充分说明我国在这一领域 的研究已达到国际一流水平。

在利用分子和形态联合数据方面, 我国科学家 也对一些重要植物类群开展了系统学和分类学研 究，取得了具有世界影响的成果。例如，Wang等 (2009)利用 4 个DNA片段和 65 个广义形态学形状在 属级水平上重建了真双子叶植物最基部的分支 毛莨目的系统发育关系，并在此系统发育框架 
下确定了各不同分类阶元的鉴别性状(diagnostic character), 从而为毛茛目提出了一个世界性的新分 类系统, 包括建立一个新族——美花草族(Callianthemeae)。这项工作是迄今在世界范围内对该目所 进行的取样最全的分子系统学研究, 对于理解毛莨 目大的进化格局和脉络, 以及整个被子植物, 特别 是真双子叶植物的多样性和花部式样的演化规律 具有重要意义。目前已被引用超过210次, 并被欧美 著名的大学教科书Plant Systematics (Michael G. Simpson著)以及被子植物系统发育网站(www.mobot. org/MOBOT/research/APweb/)引用。Yu等(2016)在 全球范围内选取了 150 种风仙花属(Impatiens)植物, 利用 46 个广义形态性状(包括种皮微形态和孢粉)和 3个DNA片段重建了该属的系统发育关系, 以此为 基础评价了形态性状的进化式样, 确定了不同支系 的鉴别性状, 从而提出了一个能反映凤仙花属进化 历史的分类系统。凤仙花属是一个超过 1,000 种的大 属, 被英国著名植物分类学家Joseph D. Hooker认 为是被子植物分类学研究中最为困难的类群。Yu等 (2016)的新系统是继Warburg和Reiche (1895)之后一 百多年来由中国学者提出的风仙花属全球性的属 下分类系统, 大大促进了凤仙花属的分类学和系统 学研究。

在系统发育基因组学方面, 我国科学家利用转 录组、简化基因组、基因组浅层测序等技术获得了 大量核基因或/和叶绿体基因, 在不同尺度上重建 了植物类群间的系统发育关系, 取得了以下成果:

(1)利用转录组数据重建了蕨类植物和真双子 叶植物大尺度的系统发育框架, 解决了基部被子植 物早期分化支系之间的系统发育关系, 澄清了倪藤 类的系统位置。这些重要成果发表在Nature Communications、GigaScience、New Phytologist和Proceedings of the Royal Society B: Biological Sciences等国 际主流刊物上, 说明我国这方面的研究已达到国际 前沿水平甚至引领了这方面的研究。

(2)利用转录组或简化基因组数据重建了植物 许多科、属的系统发育关系, 如松科、蓄微科、菊 科、十字花科、桦木科、栎属(Quercus)、马先蒿属 (Pedicularis)等。这些成果发表在Molecular Biology and Evolution、Molecular Phylogenetics and Evolution和Journal of Systematics and Evolution等国际主 流刊物上。
(3)利用叶绿体基因组数据重建了许多植物重 要代表类群的系统发育树, 如真蒝类、基部真双子 叶植物、山茱莫目、小檗科、蓄薇科、温带竹类等。 相关成果在Systematic Biology、New Phytologist、 Genome Biology and Evolution和Molecular Phylogenetics and Evolution等国际主流期刊上发表。尤其是, $\mathrm{Li}$ 等(2019)利用2,881 个质体基因组的80个基因重建 了涵盖 $85 \%$ 的现存被子植物科的高支持率的质体系 统发育树, 并估算了被子植物科级以上主要分支的 分化时间, 从而提出了被子植物化石记录与分子钟 推算时间之间的“侏罗纪空缺” (Jurassic gap)。这项 研究发表在国际植物学专业期刊Nature Plants上, 标志着我国利用质体基因组重建植物生命之树研 究已达到国际前沿水平并引领了这方面的研究。

此外，Yu等(2017)利用基因组浅层测序技术获 得了叶绿体基因组和 rDNA数据, 重建了山茶科的 系统发育关系并探讨了东亚亚热带常绿阔叶林的 进化历史。Zeng等(2018)通过文库构建流程的优化, 对25份标本(采集时间为1934-2007年不等)进行基 因组浅层测序，获得了23份标本完整或几近完整的 叶绿体基因组序列及21份标本的rDNA序列。这项 技术的成功使大量利用腊叶标本进行系统发育基 因组学研究成为可能，应用前景十分广阔。

在重建超大植物生命之树方面, 我国学者的研 究工作也很突出。例如, Wu等(2018)基于4个DNA片 段利用超矩阵的方法重建了包括55\%的䔃愁科物种 (157/285)的系统发育树，然后通过整合系统发育、 分子钟、生物地理和多样化速率分析, 发现非洲、 亚洲、澳大利亚和美洲的苼僽科谱系在中新世中晚 期都经历了快速积累，从而提出了 “全球不同大陆 旱生植物区系在中新世中晚期几乎同时兴起”的观 点。随后又结合全球㛊僽科的分布数据和气候数据, 发现水分生态位保守性和物种的快速分化共同决 定了干旱区教愁科的全球多样性分布格局。Wang 等(2018)利用 16 个DNA片段重建了包括46\%的杜鹃 花属(Rhododendron)物种(467/ 1000)的系统树, 结 合该属的全球物种分布数据, 发现热带地区杜鹃花 属物种多样性的增加是由于热带地区物种快速分 化形成的, 这与热带山区的生境异质性和气候的非 季节性相关。这些文章分别发表在BMC Evolutionary Biology、Proceedings of the Royal Society B: Biological Sciences和Global Ecology and Biogeography等国际 
主流期刊上。此外, 我国学者还在区域植物生命之 树重建方面做出了在国际上有重要影响的研究工 作。例如, Lu等(2018)利用5个DNA片段重建了包括 我国 $90 \%$ 被子植物属的生命之树(5,864个物种), 结 合分化时间和分布数据, 发现我国约 $66 \%$ 的被子植 物属是在中新世以后出现的, 在过去的 3,000 万年 中, 草本植物比木本植物经历了更加快速的分化, 且中国东部和西部植物的分化格局存在显著差异, 中国东部对草本植物起到了“博物馆”的保存效应, 对木本植物则兼具“博物馆”和“摇篮”的作用。这项 研究是中国生命之树重建研究成果第一次刊登在 国际期刊Nature上，标志着我国区域生命之树的研 究已达到国际前沿水平。

\section{3 我国在植物生命之树重建领域发展中存 问题}

尽管近年来我国科学家在植物生命之树重建领 域取得了重要进展，但在发展中也存在一些问题:

(1)植物系统学研究重分子而轻形态。随着植物 分子系统学的快速兴起和评价体系的引导, 越来越 多的科学家利用分子证据来重建植物类群之间的 系统发育关系, 而忽略了形态学证据。当前, 我国 已有一些植物分类学家转做分子系统学, 且不再培 养从事形态学或者经典分类学的学生, 使得从事形 态学研究的人员锐减, 研究队伍面临断层的危险。

(2)缺少国际大团队协作。我国科学家开展的绝 大多数属、种级水平的系统发育研究都集中在东亚 分布为主的植物类群上, 较少涉及世界性分布的类 群。目前, 针对一些重要的植物类群的系统发育研 究, 国际上成立了许多合作团队, 如被子植物系统 发育组(APG)、蕨类系统发育组(PPG)、禾本科系统 发育工作组(GPWG)、全球薹草组(Global Carex Group)等。这些研究团队都是外国科学家主导, 鲜 有我国学者的身影。

(3)植物系统发育基因组学分析方法过于简单。 由于我国在基因组测序技术方面已有良好的基础, 植物系统发育基因组学发展异常迅速, 很多课题组 都开始利用基因组学手段来重建植物类群间的系 统发育关系, 这将在数据分析方面对研究人员提出 更高的要求。现在国内分析基因组数据最常用的方 式还是直接多基因联合, 鲜有考虑进化饱和、谱系 间GC含量异质性、同义密码子偏选以及RNA编辑
等进化噪音对系统发育分析的影响。同时, 我国部 分学者常常报道单个被子植物物种的叶绿体基因 组, 而被子植物的叶绿体基因组结构相对比较保守, 因此这样的报道科学意义较小, 造成了一定的资源 浪费。

(4)植物分子系统学与其他学科交叉、融合较 少。我国具有完整的地层历史, 古植物学家在辽宁 西部、山东山旺、西南地区等地发现了大量的植物 化石, 为不同植物类群的起源和进化提供了关键的 证据。然而, 古植物研究团队和分子系统学研究团 队往往各自占据一个研究领域，缺少跨领域实质性 的合作。直接的后果是我国在这两个领域都取得了 很好的进展，但还不足以形成新的理论。虽然在重 建超大植物生命之树方面有所尝试, 并取得了一些 不错的进展，但在利用超大生命之树探讨生物多样 性的进化动态和分布格局的成因等方面研究较少。 一方面, 与国外相比, 我国科学家在大数据分析和 软件开发与应用方面还存在较大差距; 另一方面, 缺乏和生态学家、古气候学家的合作。

(6)植物类群研究缺乏系统性。从近几届“全国 系统与进化植物学研讨会暨青年学术研讨会”的参 会规模看, 我国的植物生命之树重建的研究队伍是 比较大的, 但在国际上具有广泛影响力的科学家较 少。同时, 不同研究团队之间研究的类群缺乏联系, 各自为战, 难以形成体系。这些因素导致我国在国 际竞争中受到明显的制约。

\section{4 对我国植物生命之树重建未来发展的建议}

针对我国植物生命之树重建领域目前存在的 问题，我们提出以下几个方面的建议:

(1)巩固现有重要植物类群生命之树重建的研 究体系。生物多样性在不同地区、不同支系的分布 都是极其不平衡的。探讨生物多样性的起源和时空 格局是进化生物学家和生态学家共同关心的基本 科学问题。联合多基因和形态数据为植物类群建立 合理自然的分类系统; 应用基因组数据解决少数基 因数据难以解决的快速辐射等支系的系统发育问 题; 通过整合系统学、形态学、古生物学、生态学 等多学科的手段、方法, 揭示生物多样性的进化动 态和促进因子，阐明其时空格局及其成因。

(2)不同研究方向使用不同的评价体系; 稳定 支持经典学科, 比如植物形态学、植物分类学, 使 
从事这些领域的研究人员没有后顾之忧, 能安心从 事研究工作, 并鼓励和支持他们培养该领域的人才 队伍。组建研究生培养指导小组来杜绝以经典学科 之名招生, 却让学生从事分子生物学相关研究现象 的发生。

(3)进行国家层面的顶层设计, 通过重大项目 促进合作和学科交叉。聚焦重要类群进行重点突破, 集中国内不同领域的优势力量, 形成“航空母舰”式 的作战体系, 使研究形成规模, 力争培养出在国际 上有影响力的大师级的科学家。

(4)在新的国际竞争中，依托我国正在实施的 “一带一路”倡议, 以及中国科学院牵头建立的中一 非联合研究中心、东南亚生物多样性研究中心、中 一乌全球苟园(昆明中心)和中国科学院华南植物园秘鲁圣马可斯大学分子系统与进化实验室等国际 合作机构, 领导和推动其他国家的科学家围绕世界 性的植物类群开展生命之树重建研究, 提升我国科 学家在植物系统学领域的国际影响力。

\section{参考文献}

APG (1998) An ordinal classification for the families of flowering plants. Annals of the Missouri Botanical Garden, 85, 531-553.

Atkinson BA (2018) The critical role of fossils in inferring deep-node phylogenetic relationships and macroevolutionnary patterns in Cornales. American Journal of Botany, 105, 1401-1411.

Bapteste E, Brinkmann H, Lee JA, Moore DV, Sensen CW, Gordon P, Duruflé L, Gaasterland T, Lopez P, Müller M, Philippe $H$ (2002) The analysis of 100 genes supports the grouping of three highly divergent amoebae: Dictyostelium, Entamoeba, and Mastigamoeba. Proceedings of the National Academy of Sciences, USA, 99, 1414-1419.

Benton MJ, Ayala FJ (2003) Dating the tree of life. Science, 300, 1698-1700.

Chase MW, Soltis DE, Olmstead RG, Morgan D, Les DH, Mishler BD, Duvall MR, Price R, Hills HG, Qiu YL, Kron KA, Retting JH, Conti E, Palmer JD, Manhart JR, Sytsma KJ, Michaels HJ, Kress WJ, Karol KG, Clark WD, Hedren M, Gaut BS, Jansen RK, Kim KJ, Wimpee CF, Smith JF, Furnier GR, Strauss SH, Xiang QY, Plunkett GM, Soltis PS, Williams SE, Gadek PA, Quinn CJ, Eguiarte LE, Golenberg E, Learn GH, Graham S, Barrett SCH, Dayanandan S, Albert VA (1993) Phylogenetics of seed plants: An analysis of nucleotide sequences from the plastid gene rbcL. Annals of the Missouri Botanical Garden, 80, 528-580.

Chen ZD, Wang XQ, Sun HY, Han Y, Zhang ZX, Zou YP, Lu AM (1998) Systematic position of the Rhoipteleaceae: Evid- ence from nucleotide sequences of the $r b c L$ gene. Acta Phytotaxonomica Sinica, 36, 1-7.

Cheng S, Melkonian M, Smith SA, Brockington S, Archibald JM, Delaux PM, Li FW, Melkonian B, Mavrodiev EV, Sun W, Fu Y, Yang H, Soltis DE, Graham SW, Soltis PS, Liu X, $\mathrm{Xu} \mathrm{X,} \mathrm{Wong} \mathrm{GK} \mathrm{(2018)} \mathrm{10KP:} \mathrm{A} \mathrm{phylodiverse} \mathrm{genome}$ sequencing plan. GigaScience, 7, giy013.

Cox CJ, Li B, Foster PG, Embley TM, Civán P (2014) Conflicting phylogenies for early land plants are caused by composition biases among synonymous substitutions. Systematic Biology, 63, 272-279.

Cronquist A (1981) An Integrated System of Classification of Flowering Plants. Columbia University Press, New York.

Darwin C (1859) The Origin of Species by Means of Natural Selection. Murray, London.

Delsuc F, Brinkmann H, Philippe H (2005) Phylogenomics and the reconstruction of the tree of life. Nature Reviews Genetics, 6, 362-375.

Doyle JA, Endress PK (2000) Morphological phylogenetic analysis of basal angiosperms: Comparison and combination with molecular data. International Journal of Plant Sciences, 161(Suppl.), 121-153.

Drew BT, Ruhfel BR, Smith SA, Moore MJ, Briggs BG, Gitzendanner MA, Soltis PS, Soltis DE (2014) Another look at the root of the angiosperms reveals a familiar tale. Systematic Biology, 63, 368-382.

Eisen JA, Fraser CM (2003) Phylogenomics: Intersection of evolution and genomics. Science, 300, 1706-1707.

Eiserhardt WL, Antonelli A, Bennett DJ, Botigué LR, Burleigh JG, Dodsworth S, Enquist BJ, Forest F, Kim JT, Kozlov AM, Leitch IJ, Maitner BS, Mirarab S, Piel WH, Pérez-Escobar OA, Pokorny L, Rahbek C, Sandel B, Smith SA, Stamatakis A, Vos RA, Warnow T, Baker WJ (2018) A roadmap for global synthesis of the plant tree of life. American Journal of Botany, 105, 614-622.

Folk RA, Sun M, Soltis PS, Smith SA, Soltis DE, Guralnick RP (2018) Challenges of comprehensive taxon sampling in comparative biology: Wrestling with rosids. American Journal of Botany, 105, 433-445.

Friis EM, Pedersen KR, Crane PR (2016) The emergence of core eudicots: New floral evidence from the earliest Late Cretaceous. Proceedings of the Royal Society B: Biological Sciences, 283, 20161325.

Gitzendanner MA, Yang Y, Wickett NJ, McKain M, Beaulieu JM (2018) Methods for exploring the plant tree of life. Applications in Plant Sciences, 6, e1039.

Glenner H, Hansen AJ, Sørensen MV, Ronquist F, Huelsenbeck JP, Willerslev E (2004) Bayesian inference of the metazoan phylogeny: A combined molecular and morphological approach. Current Biology, 14, 1644-1649.

Goremykin VV, Nikiforova SV, Biggs PJ, Zhong B, Delange P, Martin W, Woetzel S, Atherton RA, McLenachan PA, Lockhart PJ (2013) The evolutionary root of flowering 
plants. Systematic Biology, 62, 50-61.

Haeckel E (1866) Generelle Morphologie der Organismen: Allgemeine Grundzüge der Organischen Formen-Wissenschaft, Mechanisch Begründet durch die von Charles Darwin Reformirte Descendenz-Theorie. Georg Reimer, Berlin. (in German)

Hamilton JP, Buell CR (2012) Advances in plant genome sequencing. The Plant Journal, 70, 177-190.

Hennnig W (1950) Grundzüge einer Theorie der Phylogenetischen Systematik. Deutscher Zentralverlag, Berlin. (in German)

Hennnig W (1966) Phylogenetic Systematics. University of Illinois Press, Urbana, IL.

Hinchliff CE, Smith AS, Allman JF, Burleigh JG, Chaudhary R, Coghill LM, Crandall KA, Deng J, Drew BT, Gazis R, Gude K, Hibbett DS, Katz LA, Laughinghouse HD 4th, McTavish EJ, Midford PE, Owen CL, Ree RH, Rees JA, Soltis DE, Williams T, Cranston KA (2015) Synthesis of phylogeny and taxonomy into a comprehensive tree of life. Proceedings of the National Academy of Sciences, USA, 112, 12764-12769.

Jansen RK, Cai Z, Raubeson LA, Daniell H, Depamphilis CW, Leebens-Mack J, Müller KF, Guisinger-Bellian M, Haberle RC, Hansen AK, Chumley TW, Lee SB, Peery R, McNeal JR, Kuehl JV, Boore JL (2007) Analysis of 81 genes from 64 plastid genomes resolves relationships in angiosperms and identifies genome-scale evolutionary patterns. Proceedings of the National Academy of Sciences, USA, 104, 19369-19374.

Kadereit JW, Albach DC, Ehrendorfer F, Galbany-Casals M, Garcia-Jacas N, Gehrke B, Kadereit G, Kilian N, Klein JT, Koch MA, Kropf M, Oberprieler C, Pirie MD, Ritz CM, Roser M, Spalik K, Susanna A, Weigend M, Welk E, Wesche K, Zhang LB, Dillenberger MS (2016) Which changes are needed to render all genera of the German flora monophyletic? Willdenowia, 46, 39-91.

Kerkhoff AJ, Moriarty PE, Weiser MD (2014) The latitudinal species richness gradient in New World woody angiosperms is consistent with the tropical conservatism hypothesis. Proceedings of the National Academy of Sciences, USA, 111, 8125-8130.

Lavoué S (2016) Was Gondwanan breakup the cause of the intercontinental distribution of Osteoglossiformes? A time-calibrated phylogenetic test combining molecular, morphological, and paleontological evidence. Molecular Phylogenetetics and Evolution, 99, 34-43.

Lee EK, Cibrian-Jaramillo A, Kolokotronis SO, Katari MS, Stamatakis A, Ott M, Chiu JC, Little DP, Stevenson DW, McCombie WR, Martienssen RA, Coruzzi G, Desalle R (2011) A functional phylogenomic view of the seed plants. PLoS Genetics, 7, e1002411.

Lehtonen S (2011) Towards resolving the complete fern tree of life. PLoS ONE, 6, e24851.
Lewin HA, Robinson GE, Kress WJ, Baker WJ, Coddington J, Crandall KA, Durbin R, Edwards SV, Forest F, Gilbert MTP, Goldstein MM, Grigoriev IV, Hackett KJ, Haussler D, Jarvis ED, Johnson WE, Patrinos A, Richards S, Castilla-Rubio JC, van Sluys MA, Soltis PS, Xu X, Yang H, Zhang G (2018) Earth BioGenome Project: Sequencing life for the future of life. Proceedings of the National Academy of Sciences, USA, 115, 4325-4333.

Li HT, Yi TS, Gao LM, Ma PF, Zhang T, Yang JB, Gitzendanner MA, Fritsch PW, Cai J, Luo Y, Wang H, van der Bank M, Zhang SD, Wang QF, Wang J, Zhang ZR, Fu CN, Yang J, Hollingsworth PM, Chase MW, Soltis DE, Soltis PS, Li DZ (2019) Origin of angiosperms and the puzzle of the Jurassic gap. Nature Plants, 5, 461-470.

Liu L, Wu S, Yu L (2015) Coalescent methods for estimating species trees from phylogenomic data. Journal of Systematics and Evolution, 53, 380-390.

Liu Y, Cox CJ, Wang W, Goffinet B (2014) Mitochondrial phylogenomics of early land plants: Mitigating the effects of saturation, compositional heterogeneity, and codon usage bias. Systematic Biology, 63, 862-878.

Liu Y, Johnson MG, Cox CJ, Medina R, Devos N, Vanderpoorten A, Hedenäs L, Bell NE, Shevock JR, Aguero B, Quandt D, Wickett NJ, Shaw AJ, Goffinet B (2019) Resolution of the ordinal phylogeny of mosses using targeted exons from organellar and nuclear genomes. Nature Communications, 10, 1485.

Lu LM, Mao LF, Yang T, Ye JF, Liu B, Li HL, Sun M, Miller JT, Mathews S, Hu HH, Niu YT, Peng DX, Chen YH, Smith SA, Chen M, Xiang KL, Le CT, Dang VC, Lu AM, Soltis PS, Soltis DE, Li JH, Chen ZD (2018) Evolutionary history of the angiosperm flora of China. Nature, 554, 234-238.

Lu Y, Ran JH, Guo DM, Yang ZY, Wang XQ (2014) Phylogeny and divergence times of gymnosperms inferred from single-copy nuclear genes. PLoS ONE, 9, e107679.

Maddison DR (2013) The tree of life. Systematic Biology, 62, 179.

MaddisonWP, Midford PE, Otto SP (2007) Estimating a binary character's effect on speciation and extinction. Systematic Biology, 56, 701-710.

McKain MR, Johnson MG, Uribe-Convers S, Eaton D, Yang Y (2018) Practical considerations for plant phylogenomics. Applications in Plant Sciences, 6, e1038.

Medina R, Johnson M, Liu Y, Wilding N, Hedderson TA, Wickett N, Goffinet B (2018) Evolutionary dynamism in bryophytes: Phylogenomic inferences confirm rapid radiation in the moss family Funariaceae. Molecular Phylogenetetics and Evolution, 120, 240-247.

Moore MJ, Bell CD, Soltis PS, Soltis DE (2007) Using plastid genome-scale data to resolve enigmatic relationships among basal angiosperms. Proceedings of the National Academy of Sciences, USA, 104, 19363-19368.

Moore MJ, Dhingra A, Soltis PS, Shaw R, Farmerie WG, Folta 
KM, Soltis DE (2006) Rapid and accurate pyrosequencing of angiosperm plastid genomes. BMC Plant Biology, 6, 17.

Moore MJ, Soltis PS, Bell CD, Burleigh JG, Soltis DE (2010) Phylogenetic analysis of 83 plastid genes further resolves the early diversification of eudicots. Proceedings of the National Academy of Sciences, USA, 107, 4623-4628.

Morales-Briones DF, Liston A, Tank DC (2018) Phylogenomic analyses reveal a deep history of hybridization and polyploidy in the Neotropical genus Lachemilla (Rosaceae). New Phytologist, 218, 1668-1684.

Moreira D, Le Guyader H, Philippe H (2000) The origin of red algae and the evolution of chloroplasts. Nature, 405, 69-72.

Murphy WJ, Eizirik E, Johnson WE, Zhang YP, Ryder OA, O’Brien SJ (2001) Molecular phylogenetics and the origins of placental mammals. Nature, 409, 614-618.

Nandi O, Chase MW, Endress PK (1998) A combined cladistic analysis of angiosperms using $r b c L$ and non-molecular data sets. Annals of the Missouri Botanical Garden, 79, 249-265.

Nixon KC (1996) Paleobotany in cladistics and cladistics in paleobotany: Enlightenment and uncertainty. Review of Palaeobotany and Palynology, 90, 361-373.

One Thousand Plant Transcriptomes Initiative (2019) One thousand plant transcriptomes and the phylogenomics of green plants. Nature, 574, 679-685.

O’Reilly JE, Puttick MN, Pisani D, Donoghue PCJ (2018) Probabilistic methods surpass parsimony when assessing clade support in phylogenetic analyses of discrete morphological data. Palaeontology, 61, 105-118.

Palmer JD, Soltis DE, Chase MW (2004) The plant tree of life: An overview and some points of view. American Journal of Botany, 91, 1437-1445.

Pennisi E (2017) Sequencing all life captivates biologists. Science, 355, 894-895.

Pyron RA (2011) Divergence time estimation using fossils as terminal taxa and the origins of Lissamphibia. Systematic Biology, 60, 466-481.

Qiu YL, Lee J, Bernasconi-Quadroni F, Soltis DE, Soltis PS, Zanis M, Zimmer EA, Chen Z, Savolainen V, Chase MW (1999) The earliest angiosperms: Evidence from mitochondrial, plastid and nuclear genomes. Nature, 402, 404-407.

Rothwell GW, Escapa IH, Tomescu AMF (2018a) Tree of death: The role of fossils in resolving the overall pattern of plant phylogeny. American Journal of Botany, 105, 1239-1242.

Rothwell GW, Millay MA, Stockey RA (2018b) Resolving the overall pattern of marattialean fern phylogeny. American Journal of Botany, 105, 1304-1314.

Schneider H, Schuettpelz E (2016) Systematics and evolution of lycophytes and ferns. Journal of Systematics and Evolution, 54, 561-562.

Schrago CG, Aguiar BO, Mello B (2018) Comparative evaluation of maximum parsimony and Bayesian phylogenetic reconstruction using empirical morphological data. Journal of Evolutionary Biology, 31, 1477-1484.

Smith SA, Beaulieu JM, Donoghue MJ (2009) Mega-phylogeny approach for comparative biology: An alternative to supertree and supermatrix approaches. BMC Evolutionary Biology, 9, 37.

Smith SA, Brown JW, Yang Y, Bruenn R, Drummond CP, Brockington SF, Walker JF, Last N, Douglas NA, Moore MJ (2018) Disparity, diversity, and duplications in the Caryophyllales. New Phytologist, 217, 836-854.

Smith SA, Donoghue MJ (2008) Rates of molecular evolution are linked to life history in flowering plants. Science, 322, 86-89.

Soltis DE, Moore MJ, Burleigh G, Soltis PS (2009) Molecular markers and concepts of plant evolutionary relationships: Progress, promise and future prospects. Critical Reviews in Plant Sciences, 28, 1-15.

Soltis DE, Moore MJ, Sessa EB, Smith SA, Soltis PS (2018) Using and navigating the plant tree of life. American Journal of Botany, 105, 287-290.

Stull GW, Schori M, Soltis DE, Soltis PS (2018) Character evolution and missing (morphological) data across Asteridae. American Journal of Botany, 105, 470-479.

Su JX, Wang W, Zhang LB, Chen ZD (2012) Phylogenetic placements of two enigmatic genera, Borthwickia and Stixis, based on molecular and pollen data and description of a new family of Brassicales, Borthwickiaceae. Taxon, 61, 601-611.

Swofford DL, Waddell PJ, Huelsenbeck JP, Foster PG, Lewis PO, Rogers JS (2001) Bias in phylogenetic estimation and its relevance to the choice between parsimony and likelihood methods. Systematic Biology, 50, 525-539.

Takhtajan AL (1969) Flowering Plants: Origin and Dispersal. Oliver and Boyd, Edinburgh.

Takhtajan AL (2009) Flowering Plants, 2nd edn. Springer, Heidelberg.

Thornhill AH, Baldwin BG, Freyman WA, Nosratinia S, Kling MM, Morueta-Holme N, Madsen TP, Ackerly DD, Mishler BD (2017) Spatial phylogenetics of the native California flora. BMC Biology, 15, 96.

Twyford AD (2018) The road to 10,000 plant genomes. Nature Plants, 4, 312-313.

Wagner WH (1952) The fern genus Diellia: Structure, affinities, and taxonomy. University of California Publications in Botany, 26, 1-212.

Wang Q, Wu S, Su X, Zhang L, Xu X, Lyu L, Cai H, Shrestha N, Liu Y, Wang W, Wang Z (2018) Niche conservatism and elevated diversification shape species diversity in drylands: Evidence from Zygophyllaceae. Proceedings of the Royal Society B: Biological Sciences, 285, 20181742.

Wang W, Liu Y, Yu SX, Gao TG, Chen ZD (2013) Gymnaconitum, a new genus of Ranunculaceae endemic to the Qinghai-Tibetan Plateau. Taxon, 62, 713-722.

Wang W, Lu AM, Ren Y, Endress ME, Chen ZD (2009) 
Phylogeny and classification of Ranunculales: Evidence from four molecular loci and morphological data. Perspectives in Plant Ecology, Evolution and Systematics, 11, 81-110.

Wang W, Ortiz RDC, Jacques FMB, Liu Y, Xiang XG, Yu SX, Chen ZD (2017) New insights into the phylogeny of Burasaieae (Menispermaceae) with the recognition of a new genus and emphasis on the southern Taiwanese and mainland Chinese disjunction. Molecular Phylogenetetics and Evolution, 109, 11-20.

Wang W, Zhang XX, Chen ZD, Lu AM (2017) Comments on the APG's classification of angiosperms. Biodiversity Science, 25, 418-426. (in Chinese with English abstract) [王 伟, 张晓霞, 陈之端, 路安民 (2017) 被子植物APG分类 系统评论. 生物多样性, 25, 418-426.]

Warburg O, Reiche K (1895) Balsaminaceae. In: Die Natürlichen Pflanzenfamilien (eds Engler HGA, Prantl KAE), pp. 383-392. Wilhelm Engelmann, Leipzig. (in German)

Wen J, Liu J, Ge S, Xiang QY (Jenny), Zimmer EA (2015) Phylogenomic approaches to deciphering the tree of life. Journal of Systematics and Evolution, 53, 369-370.

Wickett NJ, Mirarab S, Nguyen N, Warnow T, Carpenter E, Matasci N, Ayyampalayam S, Barker MS, Burleigh JG, Gitzendanner MA, Ruhfel BR, Wafula E, Der JP, Graham SW, Mathews S, Melkonian M, Soltis DE, Soltis PS, Miles NW, Rothfels CJ, Pokorny L, Shaw AJ, DeGironimo L, Stevenson DW, Surek B, Villarreal JC, Roure B, Philippe H, dePamphilis CW, Chen T, Deyholos MK, Baucom RS, Kutchan TM, Augustin MM, Wang J, Zhang Y, Tian Z, Yan Z, Wu X, Sun X, Wong GK, Leebens-Mack J (2014) Phylotranscriptomic analysis of the origin and early diversification of land plants. Proceedings of the National Academy of Sciences, USA, 111, E4859-E4868.

Woese CR, Fox GE (1977) Phylogenetic structure of the prokaryotic domain: The primary kingdoms. Proceedings of the National Academy of Sciences, USA, 74, 5088-5090.

Wu SD, Zhang LJ, Lin L, Yu SX, Chen ZD, Wang W (2018) Insights into the historical assembly of global dryland floras: the diversification of Zygophyllaceae. BMC Evolutionary Biology, 18, 166.

Xi Z, Ruhfel BR, Schaefer H, Amorim AM, Sugumaran M, Wurdack KJ, Endress PK, Matthews ML, Stevens PF, Mathews S, Davis CC (2012) Phylogenomics and a posteriori data partitioning resolve the Cretaceous angiosperm radiation Malpighiales. Proceedings of the National Academy of
Sciences, USA, 109, 17309-17310.

Xiang XG, Xiang KL, Ortiz RDC, Jabbour F, Wang W (2019) Integrating palaeontological and molecular data reveals ancient and recent dispersal patterns in the pantropical Hamamelidaceae. Journal of Biogeography, 46, 1622-1631.

Yonezawa T, Segawa T, Mori H, Campos PF, Hongoh Y, Endo H, Akiyoshi A, Kohno N, Nishida S, Wu J, Jin H, Adachi J, Kishino H, Kurokawa K, Nogi Y, Tanabe H, Mukoyama H, Yoshida K, Rasoamiaramanana A, Yamagishi S, Hayashi Y, Yoshida A, Koike H, Akishinonomiya F, Willerslev E, Hasegawa M (2017) Phylogenomics and morphology of extinct paleognaths reveal the origin and evolution of the Ratites. Current Biology, 27, 68-77.

Yu SX, Janssens SB, Zhu XY, Lidén M, Gao TG, Wang W (2016) Phylogeny of Impatiens (Balsaminaceae): Integrating molecular and morphological evidence into a new classification. Cladistics, 32, 179-197.

Yu XQ, Gao LM, Soltis DE, Soltis PS, Yang JB, Fang L, Yang SX, Li DZ (2017) Insights into the historical assembly of East Asian subtropical evergreen broadleaved forests revealed by the temporal history of the tea family. New Phytologist, 215, 1235-1248.

Zanne AE, Tank DC, Cornwell WK, Eastman JM, Smith SA, FitzJohn RG, McGlinn DJ, O’Meara BC, Moles AT, Reich PB, Royer DL, Soltis DE, Stevens PF, Westoby M, Wright IJ, Aarssen L, Bertin RI, Calaminus A, Govaerts R, Hemmings F, Leishman MR, Oleksyn J, Soltis PS, Swenson NG, Warman L, Beaulieu JM (2014) Three keys to the radiation of angiosperms into freezing environments. Nature, 506, 89-92.

Zeng CX, Hollingsworth PM, Yang J, He ZS, Zhang ZR, Li DZ, Yang JB (2018) Genome skimming herbarium specimens for DNA barcoding and phylogenomics. Plant Methods, 14, 43.

Zhang YJ, Li DZ (2011) Advances in phylogenomics based on complete chloroplast genomes. Plant Diversity and Resources, 33, 365-375. (in Chinese with English abstract) [张韵 洁, 李德铢 (2011) 叶绿体系统发育基因组学的研究进 展. 植物分类与资源学报, 33, 365-375.]

Zhong B, Liu L, Yan Z, Penny D (2013) Origin of land plants using the multispecies coalescent model. Trends in Plant Science, 18, 492-495.

(责任编委：高连明 责任编辑：黄祥忠) 